\title{
Layered expression scanning: multiplex analysis of RNA and protein gels
}

\author{
Michael A. Tangrea ${ }^{1}$, Michael J. Flaig ${ }^{1}$, Arun Ramesh ${ }^{2}$, Carolyn J.M. Best ${ }^{1}$, \\ Galina V. Baibakov ${ }^{2}$, Stephen M. Hewitt ${ }^{1}$, Capre D. Mitchell ${ }^{2}$, Dan-Paul Hartmann ${ }^{2,3}$, \\ Vladimir Knezevic ${ }^{2}$, Michael R. Emmert-Buck ${ }^{1}$, and Rodrigo F. Chuaqui ${ }^{1}$
}

BioTechniques 35:1280-1285 (December 2003)

Northern blots and immunoblots are utilized in laboratories worldwide and offer several important features for analyzing mRNA and protein expression, including accuracy, low cost, evaluation of probe specificity, and information on transcript and protein forms based on molecular size. However, standard blotting techniques are hampered by three factors. They require a significant amount of input material, are laborious, and are capable of measuring only one protein or transcript at a time. Here we describe a simple yet effective technique for the multiplex analysis of standard RNA and protein gels using the layered expression scanning platform. The method relies on a novel membrane with high-affinity low-capacity binding characteristics. Using this approach, multiple blots from an RNA or protein electrophoresis gel can be simultaneously produced. We believe this method will be widely applicable to expression studies for a broad range of biological systems.

\section{INTRODUCTION}

New high-throughput gene expression array $(1,2)$ and proteomic techniques $(3,4)$ identify genes (from a few dozen to several hundred) whose expression profiles provide novel insights into cellular physiology and/or allow disease states to be segregated on a molecular rather than a phenotypic basis $(5,6)$. Typically, it is necessary to validate and quantitatively measure the expression level of each gene of interest. Currently, this represents a significant challenge in terms of time and effort. Moreover, the amount of biological sample available for subsequent investigation is often limiting, particularly in the case of developmental biology samples and clinical specimens.

Layered expression scanning (LES) is a new technology codeveloped by the National Institutes of Health (Bethesda, MD, USA) and 20/20 GeneSystems (Rockville, MD, USA) (http://www.2020gene.com/) to address this next phase of research (7). The method utilizes a layered array of membranes for molecular analysis and can be applied to a variety of life science platforms, including tissue sections, cells in culture, electrophoresis gels, multiwell plates, and tissue arrays (Figure 1). The technique is performed by passing the sample through a series of membrane layers that maintain the original 2-D architecture and thus permit the concurrent measurement of different RNA transcripts or proteins in each of the individual sample elements (e.g., various cellular pheno- types in a tissue section, bands on a gel, and individual wells of a microplate). The method is conceptually simple, requires no moving parts, can be used in an open or closed format, and maintains target biomolecules at a high concentration during the analysis process to produce sensitive measurements.

To specifically facilitate the multiplex analysis of RNA and protein electrophoresis gels using LES as an open system, we developed a unique, highly efficient hybridization membrane that permits multiple blots to be produced from a single gel. Each blot can be subsequently probed for individual transcripts or proteins using standard procedures. The method increases the throughput rate of Northern blots, immunoblots, and importantly, permits an increase in the number of molecular measurements that can be made per biosample.

\section{MATERIALS AND METHODS}

\section{Multiplex Northern Blots}

Hybridization and/or total RNA binding characteristics of the layered array membranes were assessed in several different experiments as follows. Total RNA (15-30 $\mu \mathrm{g})$ from cell lines MDA-MB-453 (Geneka Biotechnology, Montreal, QC, Canada), Jurkat (Geneka Biotechnology), HeLa (Ambion, Austin, TX, USA), or osteosarcoma MG-63 (Ambion) was electrophoresed in a $1 \%$ denaturing agarose gel. After washing the gel

\footnotetext{
${ }^{1}$ National Cancer Institute, Bethesda, 220/20 GeneSystems, Rockville, MD, and

${ }^{3}$ Georgetown University Hospital, Washington, DC, USA
} 
twice for 5 min in DEPC-treated water, a standard Northern blot capillary transfer was performed overnight, except that the 10layer membrane system (20/20 GeneSystems) was substituted for a nitrocellulose membrane. The following setup was utilized (from bottom to top): $20 \times$ standard saline citrate (SSC) transfer buffer, agarose gel, 10-layer membrane set, one nitrocellulose membrane (Protran ${ }^{\circledR}$; Schleicher \& Schuell BioScience, Keene, NH, USA), 20-40 pieces of transfer paper (Gel Blot Paper; Schleicher \& Schuell BioScience), and a standardized weight $\left(7 \mathrm{~g} / \mathrm{cm}^{2}\right)$. After transfer, the membranes were UV cross-linked (1200 mJ), and total RNA capture was assessed by SYBR ${ }^{\circledR}$ DX DNA Blot Stain (Molecular Probes, Eugene, OR, USA). For experiments to analyze specific gene levels, the membranes were

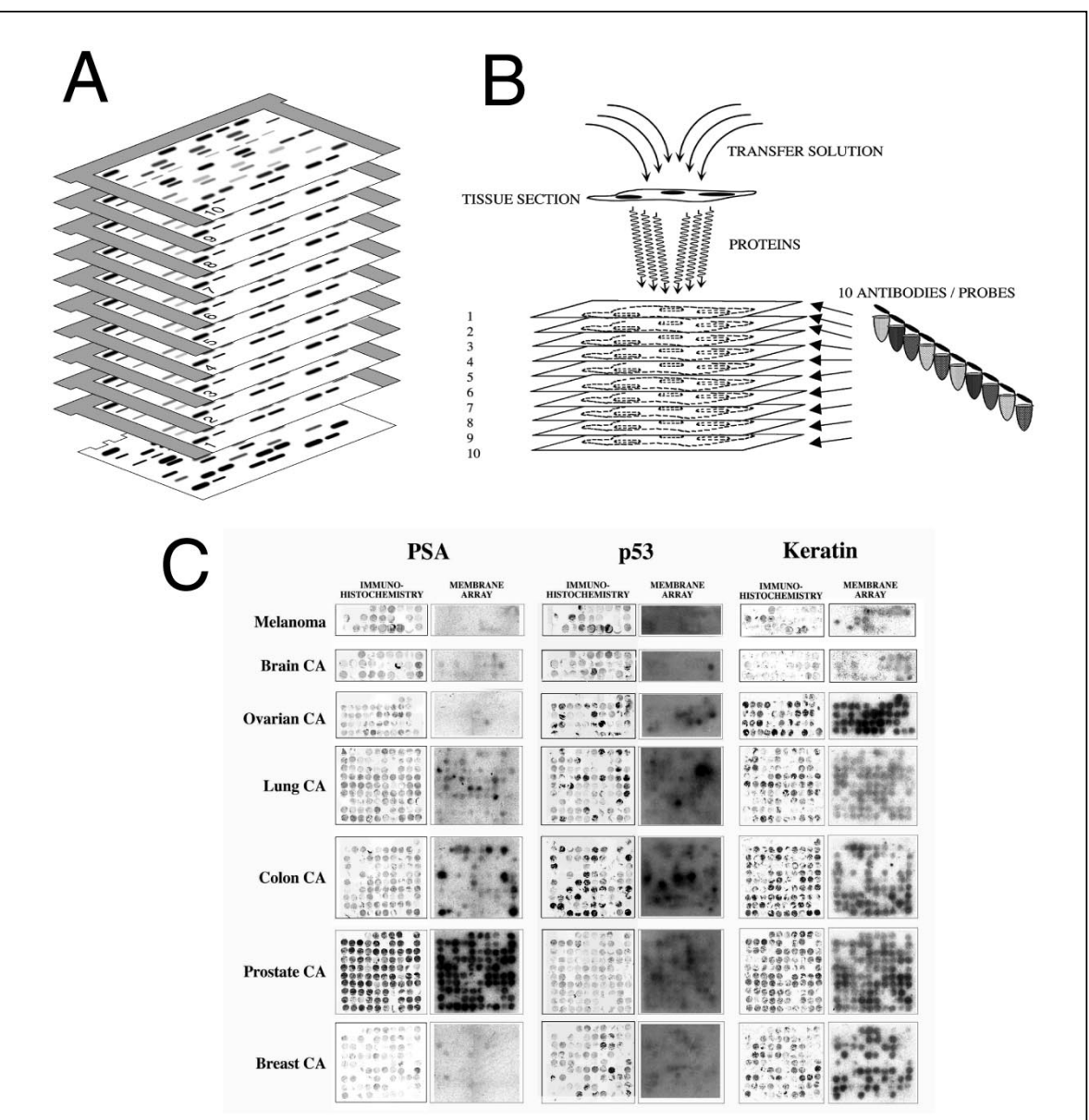

Figure 1. Applications of layered expression scanning. A biological sample is transferred through a series of analysis membranes as an intact two-dimensional (2-D) object, which allows each of the sample components to be analyzed simultaneously. The method can be utilized as either a closed system or an open system. In the open version, the membranes are specifically designed to bind a subset of the target molecules, resulting in a series of replicate membranes that can be subsequently probed for specific genes or proteins. In the closed version, specific antibodies or DNA molecules are coated onto each membrane. The sample of interest is prelabeled and passed through the layers. Proteins or mRNAs are captured by their corresponding membrane, and the expression level of each is subsequently quantified. In this application, the membranes permit nontarget molecules to pass through unimpeded; thus, the closed system can be utilized with a large number (100 or more) of layers (7). (A) Diagram illustrating the use of layered expression scanning to produce 10 blots from a single gel. This diagram was obtained from the 20/20 GeneSystems Web site. (B) Diagram showing layered membrane array analysis of a tissue section. Tissue proteins are eluted and captured on the membranes, retaining the 2-D distribution. (C) Array membranes can be created from complex biological specimens such as tissue arrays and probed for multiple molecular species of interest. CA, cancer; PSA, prostate-specific antigen. prehybridized in $6 \times \mathrm{SSC}, 0.5 \%$ sodium dodecyl sulfate (SDS), $10 \mu \mathrm{g} / \mathrm{mL}$ salmon sperm, and $5 \times$ Denhardt's solution at $55^{\circ} \mathrm{C}$ for min. A portion (25-50 ng) of each probe was random prime $55^{\circ} \mathrm{C}$ in a rotating tube overnight and then washed twice for 10 $\min$ in $0.1 \times \mathrm{SSC}, 0.5 \% \mathrm{SDS}$, twice for $10 \mathrm{~min}$ in $1 \times \mathrm{SSC}, 0.1 \%$ SDS, and exposed using the PhosphorImager ${ }^{\mathrm{TM}}$ 445SI (AmerImageQuant ${ }^{\mathrm{TM}}$ software (IQMac v1.2; Amersham Biosciences). A set of parallel experiments was also performed under identical conditions, but the stack of 10-layered membranes was replaced with a conventional nitrocellulose membrane (Protran).

\section{Multiplex Immunoblots}

The generation of multiple protein blots was first assessed by evaluating total protein staining on 10 blots. Protein extract was obtained by lysing Jurkat cells in phosphate-buffered saline (PBS) with $1 \%$ SDS. Cellular protein extract and bovine serum albumin (BSA) (both from ICN Biomedicals, Irvine, CA, USA) were separated by polyacrylamide gel electrophoresis (PAGE) on a $10 \%$ Tris-HCl gel (Bio-Rad Laboratories, Hercules, CA, USA). The transfer from the gel onto 10 membranes was performed using standard electrotransfer methods for immunoblotting. After transfer, the membranes were rinsed in double-distilled water and stained with the Fast Blue Protein Stain $^{\mathrm{TM}}$ (CHEMICON International, Temecula, CA, USA). The stained membranes were digitized by scanning on a UMAX Astra 4000 VistaScan (UMAX Technologies, Dallas, TX, USA), and the quantitation of staining intensity was performed by Kodak 1D Image Analysis Software, version 3.5 (Eastman Kodak, Rochester, NY, USA). To evaluate the total and activated levels of the Raf and Erk proteins, cellular extracts were made in PBS with $1 \%$ SDS from 8 cell lines. The samples were separated by PAGE on a $10 \%$ Tris- $\mathrm{HCl}$ gel and transferred onto 10 membranes using the BIOMAX Multi-Blot Kit for Proteins (Eastman Kodak), according to the manufacturer's recommendations. After transfer, the membranes were rinsed in Tris-buffered saline (TBS) buffer (50 mM Tris, $\mathrm{pH}$ 8.0, $150 \mathrm{mM}$ 
$\mathrm{NaCl}, 0.01 \%$ Tween ${ }^{\circledR} 20$ ) and blocked in $1 \times$ casein solution (Vector Laboratories, Burlingame, CA, USA) for $15 \mathrm{~min}$. Membranes were then incubated for $8 \mathrm{~h}$ at $40^{\circ} \mathrm{C}$ in a $1: 500 \mathrm{di}$ lution of anti-Raf antibody (Transduction Laboratories, Palo Alto, CA, USA), a 1:500 dilution of anti-phosphorylated-Raf (Cell Signaling, Beverly, CA), a 1:1000 dilution of anti-Erk antibody (Santa Cruz Biotechnology, Santa Cruz, CA, USA), or a 1:500 dilution of anti-phosphorylated-Erk antibody (Cell Signaling). After incubation, the membranes were washed in TBS buffer and incubated in a 1:2000 dilution of appropriate secondary antibody conjugated to horseradish peroxidase (HRP) (Amersham Biosciences). Proteins were visualized with ECL ${ }^{\mathrm{TM}}$ Plus reagent (Amersham Biosciences), and the images were captured on a Kodak Image Station CF440 (Eastman Kodak). After primary incubation with the antibody of interest, all of the membranes were incubated in a 1:1000 dilution of anti-GAPDH antibody (CHEMICON International) to confirm equal loading and transfer from the gel.

\section{RESULTS AND DISCUSSION}

\section{RNA Analysis}

The quantity and size distribution of RNA captured by each of the membranes in the system were determined by transferring $15 \mu \mathrm{g}$ total RNA from MDA-MB-453 cells through the layers. Figure 2A shows a typical rRNA pattern with both $28 \mathrm{~S}$ and $18 \mathrm{~S}$ bands, indicating that the low binding capacity of the membranes results in rapid saturation during the transfer process, thus permitting the majority of the sample to progress through and bind to subsequent membranes. The measurement of total RNA content for each membrane is shown in Table 1. The maximal variability was in membrane 1 , which showed a $32 \%$ increase over the average signal, and membrane 4 , which showed a $23 \%$ decrease. This degree of alteration has minimal effect on subsequent probe hybridization results and is within the normal range of the experiment-to-experiment variability typically observed with standard Northern blots.

We then evaluated the hybridization characteristics of the system by analyzing an individual gene on each of 10 membranes. Figure 2B shows a signal generated by a GAPDH probe on membranes $2,4,7$, and 8 using $15 \mu \mathrm{g}$ total RNA from the MG-63 osteosarcoma cell line. A specific $1.3-\mathrm{kb}$ band corresponding to the GAPDH transcript is seen in each membrane. Table 1 shows the quantitation of the signal. Similar to overall RNA levels, only a relatively small variation between the membranes was observed, ranging from a $41 \%$ decrease from the average to a $29 \%$ increase. Taken together, the total RNA and GAPDH quantitation data show that the layered array system generates multiple blots that can be suc-

Figure 2. RNA analysis using layered expression scanning. (A) Total RNA staining on 10 membrane layers generated from a gel with $15 \mu \mathrm{g}$ of MDAMB-453 cell line RNA. A typical rRNA pattern is seen in all membranes. (B) GAPDH signal on layers 2, 4, 7, and 8 using $15 \mu \mathrm{g}$ of osteosarcoma MG-63 cell line RNA. A 1.3-kb specific band is observed on the membranes. (C) Analysis of GAPDH (layer 2), E6/E7 (layer 4), proliferating cell nuclear antigen (PCNA) (layer 5), and cdc2 (layer 7) expression levels using $20 \mu \mathrm{g}$ of HeLa cell line total RNA.
Table 1. Inter-Membrane Signal Variability on 10-Layered Array Membranes

\begin{tabular}{|ccc|}
\hline $\begin{array}{c}\text { Membrane } \\
\text { No. }\end{array}$ & $\begin{array}{c}\text { Total RNA } \\
\text { Signal Intensity }\end{array}$ & $\begin{array}{c}\text { GAPDH Signal } \\
\text { Intensity }\end{array}$ \\
\hline 1 & 1.32 & 0.89 \\
2 & 0.89 & 0.99 \\
3 & 1.06 & 1.09 \\
4 & 0.77 & 1.29 \\
5 & 0.93 & 0.89 \\
6 & 1.07 & 0.59 \\
7 & 1.14 & 0.99 \\
8 & 1.09 & 0.79 \\
9 & 0.91 & 1.28 \\
10 & 0.83 & 1.19 \\
Total RNA and GAPDH expression were compared to the average signal \\
intensity (arbitrarily assigned as 1.0). \\
\hline
\end{tabular}

cessfully probed for a specific gene.

In addition to its low-capacity binding characteristics, the LES membrane was designed to provide increased hybridization efficiency. In other words, even though it binds signifi-

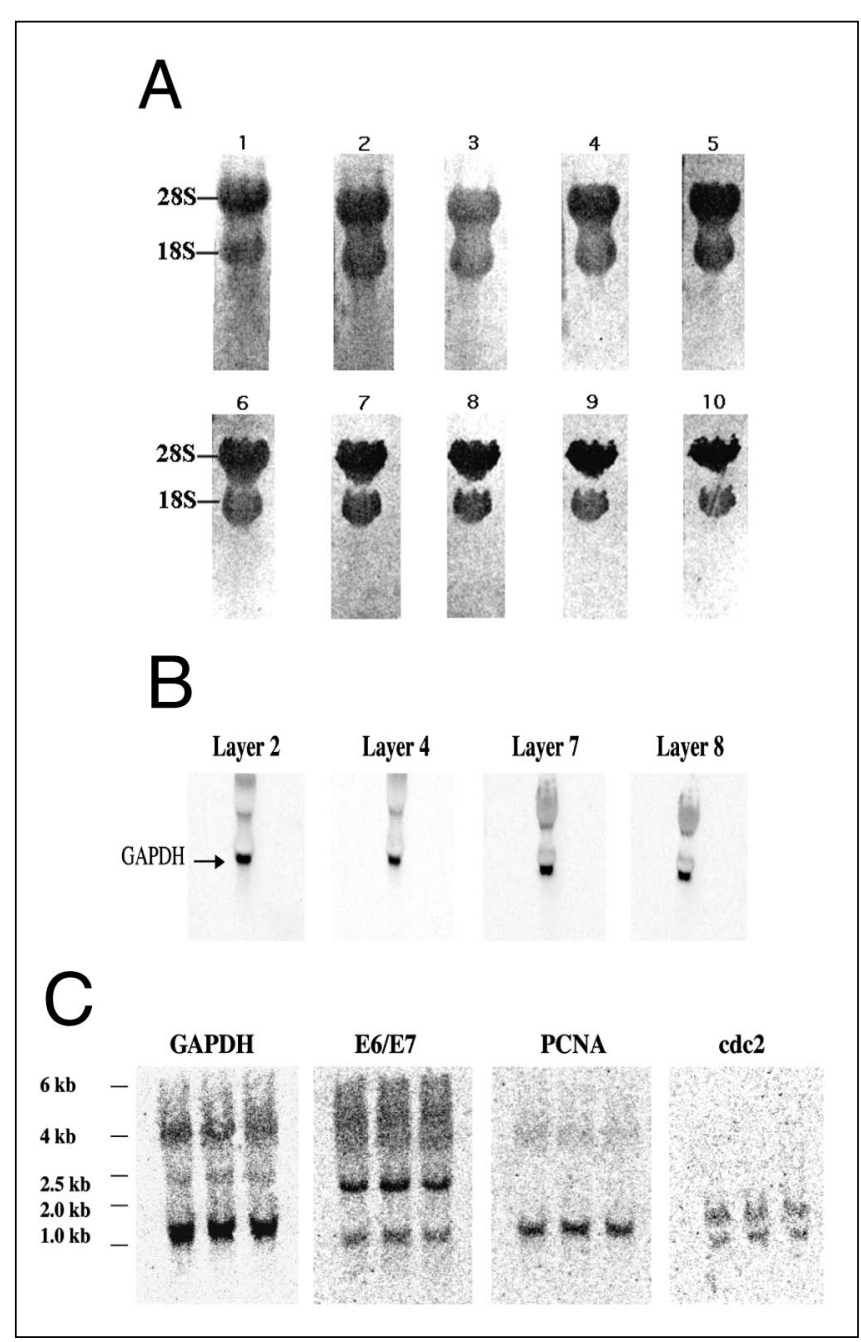


cantly less RNA or protein, the signal intensity after probing approaches that of a traditional blot. To compare hybridization results between the layered array system and a standard blot, $15 \mu \mathrm{g}$ total osteosarcoma MG-63 cell line RNA were analyzed by both methods in parallel. After the transfer of RNA from the gels, all membranes were probed, washed, and imaged under identical conditions. Qualitatively, the results were similar between the two blots in terms of specificity and hybridization background. Densitometric analysis indicated the GAPDH band on the new membrane had a signal equal to $90 \%$ of the nitrocellulose membrane. In general, we have observed that the layered array membranes produce band intensities ranging from $50 \%$ to $95 \%$ of traditional blots for both mRNA and proteins. For the membranes that show a $40 \%-50 \%$ decrease in intensity, it is necessary to expose the blots in the phosphorimager or to autoradiography film for an extended period of time to produce band patterns that are comparable to those seen on traditional blots.

To assess the uniformity and reproducibility of the layered array system, $20 \mu \mathrm{g}$ HeLa cell line total RNA were analyzed in triplicate using GAPDH, E6/E7 genes of human papillomavirus

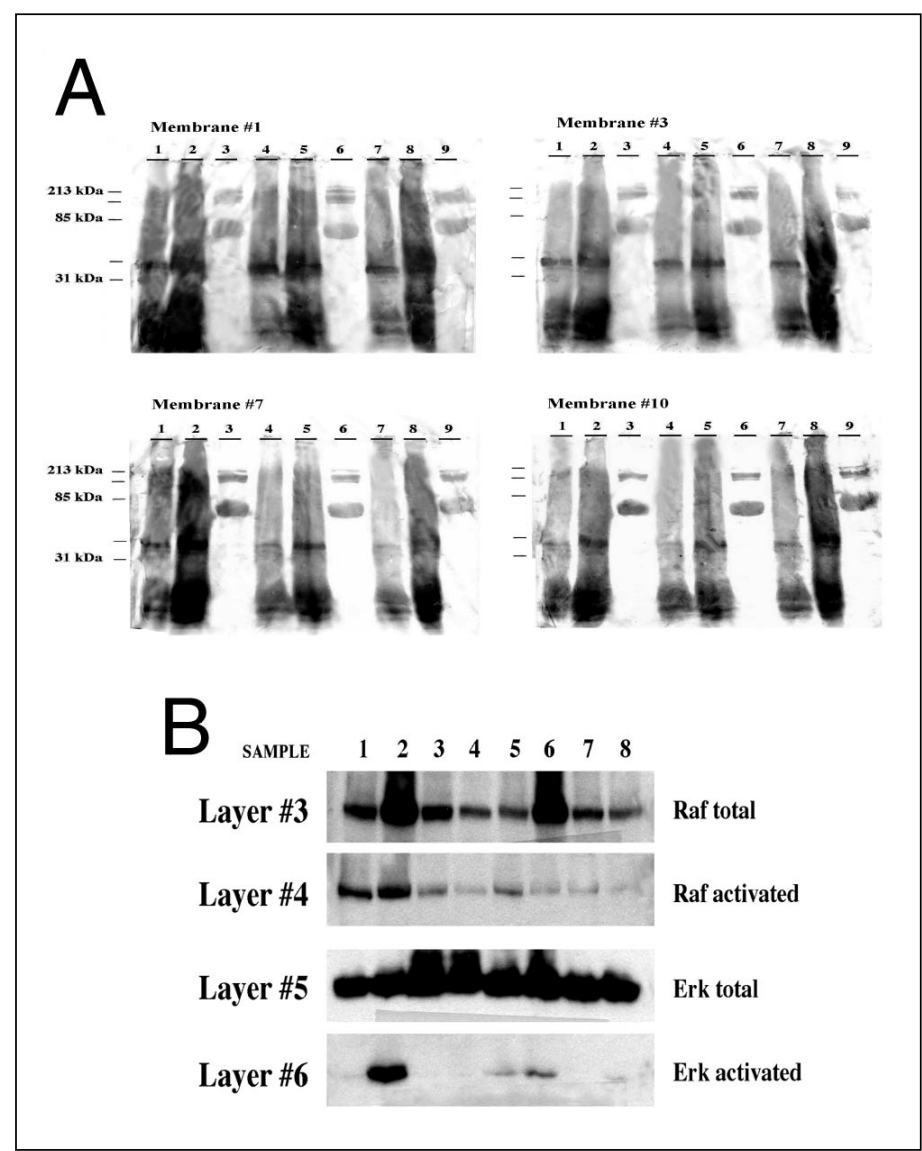

Figure 3. Protein analysis using layered expression scanning. (A) Membrane layers $1,3,7$, and 10 after the transfer of 20 (lanes 1, 4, and 7) or $40 \mu \mathrm{g}$ (lanes 2, 5, and 8) total protein lysate from Jurkat cells. Lanes 3, 6, and 9 are loaded with 10 $\mu \mathrm{g}$ bovine serum albumin (BSA). A typical total protein banding pattern is seen on the membranes. (B) Measurement of total Raf protein, phosphorylated (activated) Raf, total Erk protein, and phosphorylated (activated) Erk protein from the following eight cell lines: HaCat (lane 1), Jurkat (lane 2), HeLa (lane 3), NIH3T3 (lane 4), SW480 (lane 5), MCF7 (lane 6), 293T (lane 7), and S49 (lane 8).
(HPV) type 18, proliferating cell nuclear antigen (PCNA), and cdc2 probes (Figure 2C). The selected target genes are present in HeLa cells at varying levels of abundance, and thus the membranes could be evaluated across a 20-fold range of expression. The signals for each lane were quantified and compared (Table 2). The intramembrane lane variability ranged from a $29 \%$ increase from the average to a $19 \%$ decrease, with a median variance of $\pm 9.3 \%$. We then compared these results with the membrane variability of traditional Northern blots using nitrocellulose membranes (Tables 3 and 4). Twenty micrograms of the HeLa cell line total RNA were run on three separate electrophoresis gels and subsequently blotted onto nitrocellulose. The first blot was probed for E6/E7, the second for PCNA, and the third for cdc2. As shown in Table 3, the signal intensity varied among the lanes from a $42 \%$ percent increase from the average to a $24 \%$ decrease, with a median variance of $\pm 13.6 \%$. We also analyzed the inter membrane levels of total RNA staining on the three standard Northern blots and compared it with data from the layered array membranes (Table 1). The inter membrane total RNA levels among the three standard Northern blots varied among the lanes from a $42 \%$ percent increase from the average to a $58 \%$ decrease (Table 4 ). Therefore, based on both total RNA content and probe hybridization data, we can conclude that the layered membrane array system performs similar to standard Northern blots in terms of intra- and inter-blot reproducibility. Changes (2- to 3-fold) in expression levels could be detected using the layered technology with similar reliability, as observed using conventional techniques (data not shown).

For the layered array system, we typically probe one membrane for a housekeeping gene to normalize gel loading and then perform expression measurements of additional transcripts or proteins on the remaining layers. As a practical matter, this is an optimal use of the system and allows data to be generated quickly and efficiently. For standard Northern blots, investigators often normalize gel loading using total RNA levels in the gel. Alternatively, one can simultaneously probe a blot against a gene of interest and a housekeeping gene (if they are of different sizes) or can probe for the gene of interest, strip the blot, and reprobe for a housekeeping gene. We compared the layered array system with standard Northern blots using each of these approaches. In each instance, the layered membrane system performed similarly to standard Northern blots in accurately measuring transcript levels (data not shown).

\section{Protein Analysis}

We obtained results similar to the RNA data using protein gels. Figure 3A shows membrane layers 1, 4, 7, and 10 after the transfer of total protein lysate from Jurkat cells. The difference in intensity signal among the 10 membranes ranged from $12 \%$ to $22 \%$ from the average value. The ability to perform multiple expression measurements on the same sample preparation has significant utility for investigators. For example, different members of a signaling pathway can be simultaneously measured, including both total protein and the subset of activated protein (Figure 3B). Total protein lysate from 8 cell lines 
Table 2. Intramembrane Signal Variability on Layered Array Blots

\begin{tabular}{|ccc|}
\hline $\begin{array}{c}\text { Lane } \\
(\boldsymbol{n})\end{array}$ & Probe & $\begin{array}{c}\text { Band } \\
\text { Intensity }\end{array}$ \\
\hline 1 & E6/E7-a & 0.96 \\
2 & & 1.14 \\
3 & & 0.90 \\
1 & E6/E7-b & 1.09 \\
2 & & 1.02 \\
3 & & 0.90 \\
1 & PCNA & 0.90 \\
2 & & 1.14 \\
3 & & 0.96 \\
1 & cdc2-a & 1.14 \\
2 & & 1.05 \\
3 & & 0.81 \\
1 & cdc2-b & 0.87 \\
2 & & 1.29 \\
3 & & 0.93 \\
1 & & 0.92 \\
2 & & 0.97 \\
3 & & 1.10 \\
Each lane was compared to the average signal \\
intensity for the probe listed (arbitrarily assigned \\
as 1.0). For the HPV18 and cdc2 probes, both \\
the larger (a) and smaller (b) transcripts were \\
analyzed. PCNA, proliferating cell nuclear an- \\
tigen.
\end{tabular}

Table 3. Intramembrane Signal Variability on Standard Nitrocellulose Blots

\begin{tabular}{|ccc|}
\hline $\begin{array}{c}\text { Lane } \\
(\boldsymbol{n})\end{array}$ & Probe & $\begin{array}{c}\text { Signal } \\
\text { Intensity }\end{array}$ \\
\hline 1 & & 1.15 \\
2 & E6/E7-a & 0.91 \\
3 & & 0.94 \\
1 & & 0.91 \\
2 & E6/E7-b & 1.06 \\
3 & & 1.06 \\
1 & & 1.24 \\
2 & PCNA & 0.76 \\
3 & & 1.03 \\
1 & & 0.85 \\
2 & cdc2-a & 0.76 \\
3 & & 1.42 \\
1 & & 1.09 \\
2 & cdc2-b & 0.91 \\
3 & & 1.03 \\
Each lane was compared to the average signal \\
intensity for the probe listed (arbitrarily assigned \\
as 1.0). For the HPV18 and cddc2 probes, both \\
the larger (a) and smaller (b) transcripts were \\
analyzed. PCNA, proliferating cell nuclear an- \\
tigen.
\end{tabular}

Table 4. Inter-Membrane Signal Variability on Standard Nitrocellulose Blots

\begin{tabular}{|lcc|}
\hline & $\begin{array}{c}\text { Lane } \\
(\boldsymbol{n})\end{array}$ & $\begin{array}{c}\text { RNA Signal } \\
\text { Variability }\end{array}$ \\
\cline { 2 - 3 } Blot 1 & 1 & 1.12 \\
& 2 & 0.62 \\
Blot 2 & 3 & 1.18 \\
& 1 & 1.42 \\
& 2 & 0.42 \\
Blot 3 & 3 & 1.18 \\
& 1 & 0.82 \\
& 2 & 1.03 \\
Each lane was compared to the average signal \\
intensity of all nine lanes, and the variance \\
among the three blots was compared.
\end{tabular}

was separated by gel electrophoresis, and 10 blots were subsequently produced in a single transfer. Membranes 3-6 were analyzed for total Raf protein, phosphorylated Raf, total Erk protein, and phosphorylated Erk, respectively. The data show that the ratio of total to activated protein varies among the cell lines (see lane 6, layers 3 and 4, and lane 2, layers 5 and 6), indicating that protein expression and activation status are independently regulated. Thus, it is important to measure both forms of Raf and Erk proteins to understand the overall kinetics and activation state of the pathway. Multiplex measurements such as these are likely to become increasingly important in the future as investigators move toward a systems-based understanding of biological processes. Even when multiple measurements are not initially necessary, it is advantageous to store the membranes for future use so that additional questions can be addressed as the study progresses over time.

The LES technique offers several key advantages over other approaches that are designed for the follow-up analysis of candidate genes (8-11). All of the blots are produced from a single sample and analyzed on one gel, thus eliminating sources of potential variance such as the sample preparation and solubility in loading buffer, effects of storage and/or freezing-thawing on biomolecules, and the typical variability seen among electrophoretic gel runs.

To date, over 500 laboratories have used the BIOMAX Multi-Blot Kit for Proteins, which is marketed by Eastman Kodak, 20/20 GeneSystems, and Wako Pure Chemicals Industries, Ltd. (Osaka, Japan). The majority (65\%) of researchers were satisfied with the performance of the system.
However, the first-generation method described here does have some limitations that we are working to address. The primary complaint is the poor performance of membranes $8-10$. This is not a significant problem because most investigators prefer to utilize the 5-membrane format. However, for those interested in generating 10 measurements from each gel, we recommend probing membranes 8-10 for transcripts or proteins known to be of relatively high abundance and membranes 1-7 for gene products of unknown or lower abundance. The second most frequent complaint was a lack of sensitivity. In general, transcripts or proteins that produce a clear signal on a traditional blot will work well in the layered array system. However, in cases where the biomolecule of interest is difficult to detect on a standard blot, it will be similarly difficult (or impossible) to measure using layered membranes. This limitation is inherent to the challenges associated with accurately measuring low abundance mRNAs or proteins and is not unique to the LES system per se. Overall, feedback from the research community indicates that, similar to most laboratory techniques, the current version of the layered membrane system is highly effective for some studies and less effective for others.

In summary, we present a novel layered membrane array methodology that has immediate utility for multiplex mRNA and protein expression measurements. We anticipate this represents the first practical application of a new technique that will become valuable for the analyses of a variety of life science platforms. Additional work to improve the performance of the replicate (open) version of LES and further development of the closed platform continue in the laboratory. 


\section{REFERENCES}

1.Schena, M., D. Chalon, R.W. Davis, and P. Brown. 1995. Quantitative monitoring of gene expression patterns with a complementary DNA microarray. Science 270:467-469.

2.Velculescu, V., L. Zhang, B. Vogelstein, and K. Kinzler. 1995. Serial analysis of gene expression. Science 270:484-487.

3.Emmert-Buck, M.R., J.W. Gillespie, C.P. Paweletz, D.K. Ornstein, V. Basrur, E. Appella, Q.H. Wang, J. Huang, et al. 2000. A strategic approach for proteomic analysis of human tumors. Mol. Carcin. 27:1-8.

4.Emmert-Buck, M.R., R.L. Strausberg, D.B. Krizman, M.F. Bonaldo, R.F. Bonner, D.G. Bostwick, M.R. Brown, K.H. Buetow, et al. 2000. Molecular profiling of clinical tissue specimens: feasibility and applications. Am. J. Pathol. 156:1109-1115.

5.Alizadeh, A.A., M.B. Eisen, R.E. Davis, C. Ma, I.S. Lossos, A. Rosenwald, J.C. Boldrick, H. Sabet, et al. 2000. Distinct types of diffuse large B-cell lymphoma identified by gene expression profiling. Nature 403:503-511.

6.Golub, T.R., D.K. Slonim, P. Tamayo, C. Huard, M. Gaasenbeek, J.P. Mesirov, H. Coller, M.L. Loh, et al. 1999. Molecular classification of cancer: class discovery and class prediction by gene expression monitoring. Science 286:531-537.

7.Englert, C.R., G.V. Baibakov, and M.R. Emmert-Buck. 2000. Layered expression scanning: rapid molecular profiling of tumor samples. Cancer Res. 60:1526-1530.

8.Kononen, J., L. Bubendorf, A. Kallioniemi, M. Barlund, P. Schraml, S. Leighton, J. Torhorst, M.J. Mihatsch, et al. 1998. Tissue microarrays for high-throughput molecular profiling of tumor specimens. Nat. Med. 4:844-847.

9.Lueking, A., M. Horn, H. Eickhoff, K. Bussow, H. Lehrach, and G. Walter. 1999. Protein microarrays for gene expression and antibody screening. Anal. Biochem. 270:103-111.

10.Binz, P.A., M. Muller, D. Walther, W.V. Bienvenut, R. Gras, C. Hoogland, G. Bouchet, E. Gasteiger, et al. 1999. A molecular scanner to automate proteomic research and to display proteome images. Anal. Chem. 71:4981-4988.

11.Berndt, P., U. Hobohm, and H. Langen. 1999. Reliable automatic protein identification from matrix-assisted laser desorption/ionization mass spectrometric peptide fingerprints. Electrophoresis 20:3521-3526.

Address correspondence to Rodrigo F. Chuaqui, Laboratory of Pathology and Urologic Oncology Branch, National Cancer Institute, Rm. 2A33, Bldg. 10, 9000 Rockville Pike, Bethesda, MD 20892,USA. e-mail: chuaquir@mail.nih.gov 